\title{
CONHECIMENTO DOS PROFISSIONAIS DA SAÚDE SOBRE O DIAGNÓSTICO E CONDUTA PARA ANQUILOGLOSSIA EM BEBÊS
}

Ana Beatriz Rocha Pinto

Graduanda em Odontologia. Universidade Estadual de Maringá - UEM, Brasil.

\section{Jéssica Behrens Crispim}

Cirurgiã Dentista formada pela Universidade Estadual de Maringá. Participante do Programa de Residência da Universidade Estadual de Maringá - UEM na área de Odontopediatria, Brasil.

Thaynara de Souza Lopes

Mestranda em Odontologia Integrada, Universidade Estadual de Maringá - UEM, Brasil.

Andressa Mioto Stabile

Cirurgiã Dentista formada pela Universidade Estadual de Maringá. Participante do Programa de Residência da Universidade Estadual de Maringá - UEM na área de Odontopediatria, Brasil.

\section{Gabriela Cristina Santin}

Doutora em Odontopediatria, Faculdade de Odontologia de Ribeirão Preto, Universidade de São Paulo -USP, Brasil.

\section{Marina de Lourdes Calvo Fracasso}

Doutora em Odontopediatria. Universidade de São Paulo - USP, Brasil

Autor correspondente

Ana Beatriz Rocha Pinto anabeatrizimg@gmail.com
RESUMO: O estudo avaliou o conhecimento de profissionais da área de saúde, sobre o diagnóstico e conduta clínica para o tratamento da anquiloglossia em bebês. A amostra $(n=84)$ foi dividida em: G1 (46 médicos da ESF); G2 (13 pediatras); G3 (5 fonoaudiólogas) e G4 (20 dentistas da ESF), que responderam ao questionário (dados pessoais e aplicação do protocolo de avaliação do freio lingual, preconizado por Martinelli et al. (2012) ${ }^{6}$. Um total de 21 profissionais responderam adequadamente o questionário sendo estes $66,6 \%$ do gênero feminino, com idade média de $46,4 \pm 12,5$ anos de modo que $80,9 \%$ já receberam pacientes com queixa de anquiloglossia. Quanto à obrigatoriedade do teste da linguinha constatou-se que o Grupo 1 apresentou menor grau de informação $(57,1 \%)$ comparado ao Grupo 4 (21,4\%), no entanto $57,1 \%$ dos profissionais avaliados não o fazem rotineiramente. Referente ao protocolo, nos itens 1, 2 e 3 , três profissionais $(14,2 \%)$ assinalaram figuras com diagnóstico correto (G1 e G3); em relação ao item 4, dez profissionais $(47,6 \%)$ pontuaram corretamente. Conclui-se que houve grande desconhecimento quanto à obrigatoriedade do teste da linguinha e dificuldade no correto diagnóstico de anquiloglossia em bebês.

PALAVRAS-CHAVE: Anquiloglossia; Freio lingual; Bebês.

\section{KNOWLEDGE OF HEALTH PROFESSIONALS ON DIAGNOSIS AND TREATMENT OF ANKYLOGLOSSIA IN VERY YOUNG CHILDREN}

\begin{abstract}
Current study evaluates the knowledge of health professional on the clinical diagnosis and treatment of ankyloglossia in very young children. Samples $(n=84)$ comprised G1 (46 physicians of ESF); G2 (13 pediatric physicians); G3 (5 phonoaudiologists) and G4 (20 dentists of ESF) who answered the questionnaire (personal data and application of protocol for assessment of the short lingual frenum, following Martinelli et al. (2012). Twenty-one professionals answered adequately the questionnaires, comprising females (66.6\%), average age $46.4 \pm 12.5$ years, of whom $80.9 \%$ have already received patients with tongue-tie. In the case of the mandatory stance of the tongue test, Group 1 had the lowest information rate (57.1\%) when compared with Group 4 (21.4\%). However, $57.1 \%$ of the professionals do not do it routinely. In the case of protocol, for items 1, 2 and 3, three professional (14.2\%) marked pictures with the correct diagnosis (G1 and G3); in the case of item 4, ten professionals (47.6\%) marked correctly. Results show lack of knowledge on the mandatory stance of the ankyloglossia test and the difficulty in its correct diagnosis in very young children.
\end{abstract}

KEY WORDS: Ankyloglossia; Lingual frenum; Very young children. 


\section{INTRODUÇÃO}

A língua é um órgão especializado, localizado na cavidade bucal, participa ativamente nas funções de sucção, deglutição, mastigação e fala. Possui, em sua face inferior, uma pequena prega de membrana mucosa que a conecta ao assoalho da boca, sendo denominada frênulo da língua ${ }^{1,2}$.

Em alguns casos esse tecido pode estar inserido de forma anômala, pela falha no processo de apoptose dessa estrutura durante a embriogênese sendo essa uma alteração congênita denominada anquiloglossia, comumente denominada de "língua presa"1,2,4. A anquiloglossia pode ser classificada de acordo com os aspectos anatômicos do frênulo lingual, sendo este: (1) curto - quando seu comprimento é menor que o padrão; (2) anteriorizado - quando se encontra posicionado muito próximo ao ápice da língua e (3) curto e anteriorizado - apresentando as duas características descritas anteriormente ${ }^{2,3}$.

A incidência dessa anomalia tem sido bastante estudada e pesquisas indicam que a porcentagem de crianças acometidas varia de $0,88 \%$ e $12,8 \% 1,2,3,5$. Tal variação explica-se pela diversidade de fundamentos utilizados pelos profissionais para diagnosticar e classificar a anomalia, tanto anatomicamente quanto funcionalmente ${ }^{6}$. Diferenciar essas variações requer conhecimento da anatomia da língua e do assoalho da boca para identificar se esses achados podem comprometer a movimentação da língua e as funções orofaciais ${ }^{4}$. O frênulo lingual alterado traz consigo uma série de consequências ao recém-nascido, comprometendo funções fundamentais como a sucção que, por sua vez, interfere diretamente na amamentação, culminando na perda de peso excessiva e no desmame precoce. Além disso, o movimento inadequado de sucção do bebê provoca desconforto para a mãe durante as mamadas $^{1,2,5,7}$.

A permanência do frênulo lingual anormal após esse período também traz prejuízos relacionados à dicção, à mastigação, à deglutição, ao desenvolvimento das dentições, podendo causar eventuais problemas sociais $^{2,5}$. Frequentemente observam-se dificuldades relacionadas à fala uma vez que determinados movimentos podem limitar abertura de boca e, consequentemente, os seus grupos consonatais ${ }^{1,8}$. Os movimentos de protrusão, retrusão, lateralização e vibração também se apresentam prejudicados?

$O$ projeto de lei $n^{\circ} 4.832 / 12$ que "obriga a realização do protocolo de avaliação do frênulo da língua em bebês, em todos os hospitais e maternidades do Brasil" foi sancionado pela Presidência da República e se converteu na lei $\mathrm{n}^{\mathrm{O}} 13.002$, de 20 de junho de $2014^{10}$. Desta forma, a partir do ano de 2015, passou a ser obrigatória, na rede pública de saúde, a realização do "Teste da Linguinha", que prevê que ao nascimento, o recém-nascido deverá ser avaliado clinicamente com o objetivo de constatar previamente a anquiloglossia e se necessário for indicar a cirurgia, evitando complicações futuras ${ }^{11,12}$.

O procedimento para correção do frênulo é denominado de frenectomia e consiste em uma técnica cirúrgica que tem como objetivo seccionar o freio. A técnica é simples, podendo ser realizada com anestésico tópico ou mesmo com anestesia infiltrativa, apresentando pouco ou nenhum sangramento e o mínimo de desconforto para a criança recém-nascida ${ }^{5,9}$. A conduta do diagnóstico e tratamento pode ser orientada por diversos profissionais da saúde como o médico pediatra, odontopediatra e fonoaudiólogo. Entretanto, alguns desses profissionais não estão devidamente capacitados para a realização de um adequado diagnóstico e correção cirúrgica adequada da anquiloglossia ${ }^{4,6}$.

Assim, tendo em vista a recente implementação da obrigatoriedade do "teste da linguinha" e a dificuldade da padronização do reconhecimento da anquiloglossia pelos profissionais da área da saúde, o objetivo deste estudo foi avaliar o conhecimento de profissionais da área de saúde, com respeito ao diagnóstico e conduta clínica para o tratamento do freio lingual em bebês.

\section{MATERIAL E MÉTODOS}

O presente trabalho foi submetido ao Comitê Permanente de Ética em Pesquisa Envolvendo Seres Humanos da Universidade Estadual de Maringá, parecer número 1.375.823/2015(CAAE 48165315.6.0000.0104).

Foi realizado um estudo transversal com a população de profissionais da rede pública de saúde do município de Maringá, região Noroeste do Paraná, sul do Brasil. 
O município de Maringá possui atualmente cerca de 400.000 habitantes e Índice de Desenvolvimento $\mathrm{Hu}$ mano Municipal (IDHM) de $0,808^{13}$. A rede de saúde de Maringá conta hoje com 35 Unidades Básicas de Saúde (UBS), duas Unidades de Pronto Atendimento (UPA), um Hospital Universitário (HUM/UEM) e nove hospitais privados. A Estratégia Saúde da Família (ESF) compõe-se de 61 equipes, com cobertura de $80 \%$ da população. No município nascem aproximadamente 7 mil crianças por ano, sendo esse um dado relevante ao se considerar a obrigatoriedade do "teste da linguinha" ao recém-nascido ${ }^{14}$.

Para compor a amostra do presente estudo foram incluídos todos os profissionais da área da saúde do Programa Estratégia Saúde da Família prestadores de serviço no Sistema Único de Saúde (SUS) do município de Maringá $(\mathrm{n}=84)$, divididos em quatro grupos: G1 - 46 médicos da Estratégia Saúde da Família, G2 - 28 médicos pediatras, G3 - cinco fonoaudiólogas e G4- 20 cirurgiões-dentista da Estratégia Saúde da Família.

Estes profissionais foram entrevistados pela primeira autora, por meio de um questionário semiestruturado, no local e horário de trabalho do profissional, após assinatura do termo de consentimento.

$\mathrm{Na}$ primeira etapa do questionário os profissionais preencheram dados correspondentes aos seus dados pessoais, sexo, idade e tempo em que reside na cidade de Maringá. Também foram colhidos dados profissionais a respeito do tempo de formação do profissional bem como de atuação na rede pública de saúde; área de especialização; e atendimento frequente às crianças. Além disso, questionou-se a respeito da queixa frequente de anquiloglossia na prática clínica e o conhecimento da lei $\mathrm{n}^{\mathrm{o}} 13.002$ e obrigatoriedade do "Teste da Linguinha" ao nascimento e, ainda, se o profissional possui o hábito de realizá-lo.

Na segunda etapa os profissionais responderam a um questionário, baseado no protocolo clínico preconizado por Martinelli et al. (2012) ${ }^{6}$, utilizando-se somente a parte I do protocolo, referente ao exame clínico. Por meio das figuras ilustrativas disponibilizadas no protocolo, demonstraram-se as diferentes situações para o diagnóstico e tratamento do frênulo lingual, divididos em quatro tópicos, atribuindo-se escores para cada situação. Desta forma, coube ao profissional assinalar com um X, dentre as alternativas expostas e abaixo de cada figura apresentada, os dados referentes a área anátomofuncional das estruturas da boca do bebê, como se segue:

Tópico 1- postura dos lábios em repouso: em repouso (0), entreabertos (1) ou abertos (1);

Tópico 2- tendência do posicionamento da língua durante o choro: na linha média (0), elevada (0), na linha média com elevação das laterais (2) ou baixa (2);

Tópico 3- forma da ponta da língua quando elevada durante o choro: arredondada (0), ligeira fenda no ápice (2), formato de coração (3);

Tópico 4- frênulo da língua: se é possível visualizar; não é possível visualizar ou visualizado com manobra;

Tópico 4.1- espessura do frênulo: delgado (0) ou espesso (2);

Tópico 4.2- fixação do frênulo na face sublingual da língua: no terço médio (0), entre o terço médio e o ápice (2) ou no ápice (3);

Tópico 4.3- fixação do frênulo no assoalho de boca: visível a partir das carúnculas sublinguais $(0)$ ou visível a partir da crista alveolar inferior (1).

De posse do questionário respondido pelo profissional, foram atribuídas a ele as pontuações indicativas de anormalidade do frênulo lingual, como preconizado por Martinelli et al. (2012). Na avaliação anátomo-funcional, quando a soma dos itens 1,2 e 3 for igual ou maior que 4, pode-se considerar a interferência do frênulo na movimentação da língua. Para estabelecer o valor mínimo de 4 pontos foram considerados como itens de alteração: língua baixa ou na linha média com elevação das laterais (2) e ligeira fenda no ápice (2).

Ainda na avaliação anátomo-funcional, quando a soma do item 4 for igual ou maior que 3 pode-se considerar a interferência do frênulo lingual na movimentação da língua. Para estabelecer o valor mínimo de 3 pontos foram considerados como itens de alteração: frênulo espesso e fixação do frênulo no assoalho da boca visível a partir da crista alveolar inferior, com valores 2 e 1 , respectivamente, de acordo com o score proposto no protocolo em questão.

Total da avaliação anátomo-funcional (Item 1, 2, 3 e 4): melhor resultado $=\mathrm{a} 0$ e pior resultado $=\mathrm{a} 6$. Em escala progressiva de pontuação, o score 0 significa a 
normalidade do freio lingual, enquanto a pontuação um e dois, em ordem crescente, indicam características de alteração.

Os dados colhidos foram examinados e transcritos para um banco de dados no Excel 2007 - Microsoft Corporation e submetidos a uma análise descritiva, obtendo-se a frequência absoluta (\%) das variáveis estudadas.

\section{RESULTADOS}

No presente estudo, da totalidade dos protocolos enviados aos profissionais $(n=84)$, somente 21 (25\%) foram devolvidos devidamente respondidos, de acordo com a seguinte distribuição: G1 - 46 médicos da Estratégia Saúde da Família, com nove respostas (19,6\%), G2 - 13 médicos pediatras, com quatro respostas $(30,76 \%)$, G3 - cinco fonoaudiólogas, somente um retorno (21\%) e G4- 20 cirurgiões-dentista do Programa Saúde da Família, com sete protocolos respondidos (35\%). (Figura 1)

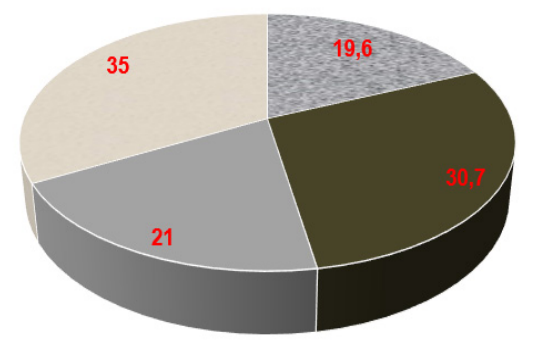

-G1-Médicos do ESF · G2-Médico Pediatra - G3-Fonoaudiólogo = G4-Dentista do ESF

Figura 1. Análise descritiva (\%) dos profissionais participantes do estudo que responderam ao questionário.

No que se refere ao perfil da amostra, constatou-se que 66,6\% dos profissionais avaliados são do gênero feminino, média de idade de 46,4 $\pm 12,5$ anos, aproximadamen-


e que, destes $95,2 \%$ são pós-graduados. Todos os profissionais são residentes em Maringá, com uma média de $21,6 \pm 13,9$ anos de residência no município e estão inse-

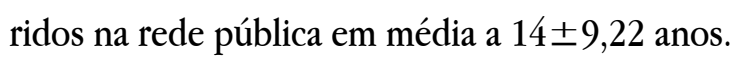

Entre os profissionais avaliados $90,4 \%$ relataram realizar frequentemente atendimento a crianças na Unidade Básica de Saúde. Além disso, 80,9\% de todos os profissionais avaliados já receberam pacientes com queixa de anquiloglossia, dentre eles destacam-se os profissionais cirurgiões-dentistas e médicos, totalizando $70,4 \%$ da amostra coletada.

Em relação ao conhecimento sobre a lei $\mathrm{n}^{\mathrm{0}} 13.002^{10}$ que instituiu o "Teste da Linguinha", obrigatório em recém-nascidos desde 2014 na rede pública e privada, apenas $33,3 \%$ relataram ter conhecimento. Dentre os protocolos avaliados, observou-se que o G1 (médicos da Estratégia Saúde da Família) apresentou menor grau de informação quanto à obrigatoriedade do procedimento $(57,1 \%)$, quando comparado ao G4 $(21,4 \%)$.

Quanto à conduta clínica dos profissionais em realizar o exame da cavidade bucal para inspeção do freio lingual, 57,1\% fazem a avaliação rotineiramente no atendimento aos bebês ( 6 médicos do ESF; 3 pediatra, 1 fonoaudióloga e 2 cirurgiões-dentistas) como mostrado na Figura 2.

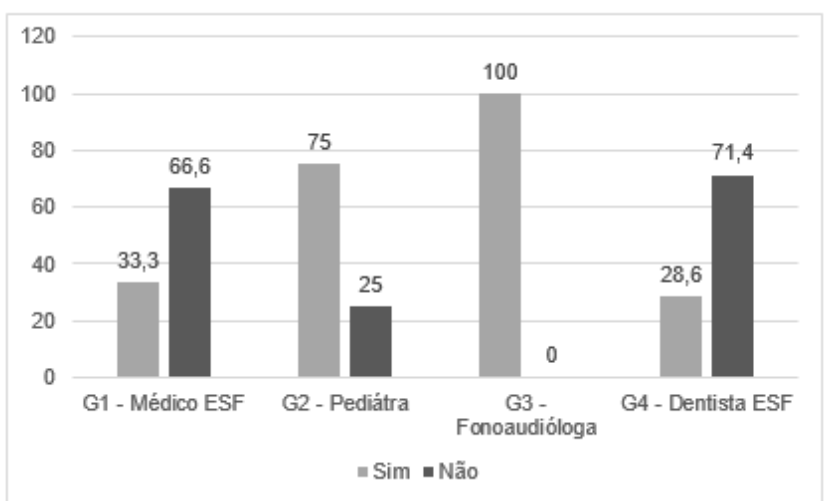

Figura 2. Análise descritiva (frequência relativa) dos profissionais participantes do estudo que realizam a avaliação do freio lingual dos bebês nas consultas de rotina, apresentado por categoria profissional.

Quando analisados os dados da segunda etapa da pesquisa, referente ao protocolo e avaliação anátomo-funcional, itens 1,2 e 3 , somente três profissionais $(14,2 \%)$, dos G1 e G3, assinalaram as figuras que equivalem a respostas com pontuação inferior a quatro, ou seja, um diagnóstico correto para a avaliação, Figura 3. 




Figura 3. Análise descritiva (n) das respostas dos profissionais participantes do estudo, em relação à avaliação anátomo-funcional (item $1,2$ e 3$)$.

No item 2, que avalia o posicionamento da língua durante o choro, $57 \%$ da amostra assinalaram que a língua deve estar posicionada na linha média com elevação das laterais (score 2) e 28,5\% assinalaram língua baixa (score 2), ambas as opções desfavoráveis ao posicionamento da língua.

Ainda durante o choro, 85\% dos profissionais julgaram que o formato correto da ponta da língua seria em formato de coração (score 3), destes $41 \%$ eram do G4, levando ao diagnóstico errado do posicionamento do mesmo.

Já em relação ao item 4, dez profissionais (47,6\%) apresentaram pontuação satisfatória, indicando um diagnóstico correto da anatomia do freio lingual, porém 11 profissionais $(52,3 \%)$ obtiveram pontuação igual ou superior a 4, demonstrando desconhecimento da anatomia do freio lingual, Figura 4.

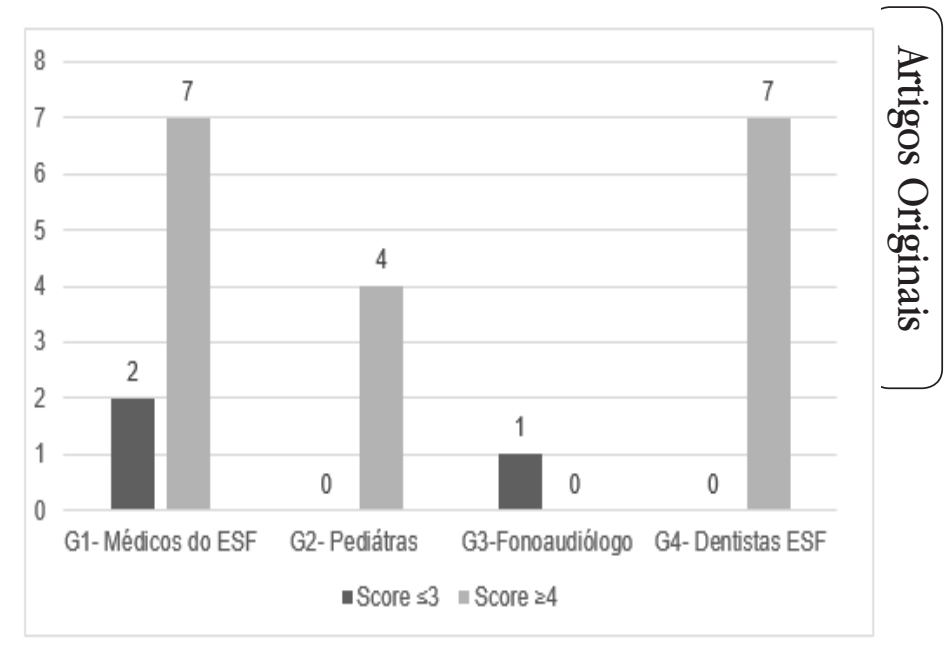

Figura 4. Análise descritiva (n) das respostas dos profissionais participantes do estudo, em relação a avaliação anátomo-funcional (item 4).

No que se refere à visualização do frênulo lingual, $71,4 \%$ dos avaliados concordam que é possível visualizá-lo sem qualquer tipo de manobra, enquanto 19\% acreditam que só é possível visualizar por meio de manobra e 9,5\% acreditam não ser possível visualizar, sendo os responsáveis por esse último dado, dois profissionais cirurgiões-dentistas.

No item seguinte, espessura do frênulo lingual alterado, $57,1 \%$ responderam delgado ao invés de espesso, sendo que a maioria das respostas insatisfatórias advém de profissionais do G1. Ainda no item em questão os cirurgiões-dentistas demonstraram melhor desempenho e conhecimento em relação à presença de anquiloglossia, sendo o grupo que melhor pontuou neste item, dentre todos os outros profissionais avaliados. Com respeito à fixação do frênulo na face ventral da língua, $47,6 \%$ assinalaram a região do ápice (3); 38\% entre o terço médio e o ápice (2), e os demais assinalaram ser no terço médio (0).

Por fim, $85,7 \%$ dos avaliados indicaram a fixação do frênulo no assoalho de boca sendo visível a partir da crista alveolar inferior (1) em detrimento das carúnculas sublinguais (0), sendo os piores resultados atribuídos aos médicos e o fonoaudiólogo. Todos os cirurgiões- dentistas bem como pediatras avaliados concordaram com a opção correta, de maior score do protocolo. 


\section{DISCUSSÃO}

A literatura científica aponta para controvérsias a respeito do correto diagnóstico de alterações na anatomia do freio lingual, denominado anquiloglossia, e dificuldades na padronização de protocolos eficazes e de fácil aplicação pelos profissionais da área da saúde ${ }^{7,15,16}$. Segundo Martinelli (2015), ${ }^{17}$ diagnosticar e diferenciar as variações anatômicas do freio lingual requer conhecimento bastante aprofundado da anatomia da língua e das áreas adjacentes para identificar se os achados anatômicos podem comprometer a movimentação da língua e, consequentemente, as funções orais.

O presente estudo constatou que houve pouca adesão dos profissionais de saúde com respeito à pesquisa, haja visto que somente 21 profissionais devolveram os questionários respondidos, dos 84 selecionados no início da pesquisa, podendo fazer uma inferência que a pouca adesão pode estar associada ao desconhecimento dos mesmos a respeito do assunto, gerando insegurança e desinteresse para o preenchimento do questionário e, ainda, outro ponto de questionamento seria a abordagem ministrada deste tema, durante o curso de graduação, nas diferentes áreas da saúde, podendo gerar um conteúdo teórico e prático insuficiente para aplicação na atividade profissional, especialmente nas Unidades Básica de Saúde.

Pesquisas científicas recentes ${ }^{15,16,17}$, com enfoque na avaliação da cavidade bucal do recém-nascido, demonstraram a importância da padronização dos procedimentos pela equipe hospitalar, atuantes no atendimento nas primeiras horas de vida, para que assim como os exames de Apgar, também, a anatomia da boca e da suas estruturas fossem avaliados, dentre eles o comprometimento funcional da língua ${ }^{6,16} \mathrm{em}$ especial o movimento de protrusão e elevação da língua durante o choro ${ }^{18}$, onde pode ser observado o formato que a língua adquire de coração ou em "V" como forma de diagnóstico de anquiloglossia ${ }^{6}$.

O diagnóstico precoce e indicação de tratamento para anquiloglossia reflete na qualidade do aleitamento materno do neonato, oferendo tratamento para as queixas por parte da mãe como dor ao amamentar e complicações na mama, fatores estes que corroboram para o desmame precoce ou desestímulo à amamentação $0^{8,16,17,19}$.
Atualmente, a importância do aleitamento materno e os prejuízos do desmame precoce já estão muito bem estabelecidos, uma vez que sua prática além de estimular o desenvolvimento adequado das estruturas orofaciais e promover um vínculo afetivo intenso entre mãe e bebê, também está ligado a indicadores de saúde materno-infantil durante toda a vida de ambos ${ }^{20,21}$.

Como segunda etapa de suporte e apoio para mãe e bebê, estão os profissionais vinculados às Unidades Básicas de Saúde, responsáveis pela puericultura deste bebê recém-nascido, já que grande parte das dificuldades com a amamentação ocorre após o retorno hospitalar, já na primeira semana. Um atendimento eficaz da equipe de saúde, apoiando mãe e bebê, para superação das dificuldades e correção das possíveis falhas, ajudarão na manutenção do aleitamento materno de qualidade.

Mais uma vez, como método auxiliar a equipe deve verificar a anatomia do freio lingual, e como ele está interferindo no processo da amamentação. Martinelli et al. $(2015)^{17}$ avaliaram o freio lingual de 109 recém-nascidos em amamentação exclusiva e quando diagnosticada a presença de anquiloglossia, a cirurgia de frenectomia foi realizada. Neste estudo concluiu-se que as crianças com indicação para a frenectomia apresentavam fadiga durante a mamada, intervalos curtos entre as mamadas e queixa de dor materna que foram solucionados após o procedimento cirúrgico bem como maior conforto às mães durante o aleitamento favorecendo sua manutenção.

No Brasil, foi promulgada, em 2014, a lei do "Teste da Línguinha" que deve compor o rol de exames obrigatórios no recém-nascido, tanto no setor público quanto privado, na tentativa de detectar a anquiloglossia precocemente. ${ }^{10}$ Neste estudo, quando os profissionais foram questionados sobre o conhecimento desta lei, somente $33,3 \%$ responderam afirmativamente, sendo que os profissionais médicos da Estratégia Saúde da Família, responsável pela grande maioria dos atendimentos clínicos nas UBSs, foram os menos informados, demonstrando que o exame da boca e estruturas anatômicas pode ser, por vezes, negligenciado. Ficou evidente que os profissionais pesquisados atendem crianças em sua área de atuação, já que a maioria relatou ter atendido crianças com queixa de anquiloglossia, mas grande parte deles não demonstrou conhecimento suficiente para avaliação correta de 
alterações do freio lingual quando responderam o questionário, assinalando figuras com diagnóstico incorreto. Mesmo os profissionais cirurgião-dentistas, habilitados e treinados para o atendimento na cavidade bucal tiveram dificuldades de associar as imagens ilustrativas a alterações do freio lingual e possível encaminhamento para a cirurgia.

Para Martinelli et al. (2012) ${ }^{6}$, a anquiloglossia pode ser facilmente identificada e diagnosticada, porém para reconhecer as variações anatômicas que o freio lingual pode apresentar, é de suma importância o conhecimento aprofundado acerca da anatomia da língua e do assoalho da cavidade oral, para que dessa forma seja possível determinar possíveis interferências nas funções orais bem como conduta e tratamento adequados.

Segundo Melo et al. $(2011)^{22}$, a frenectomia deve ser realizada de modo mais precoce possível, o que permite o retorno da mobilidade lingual, sendo uma técnica extremamente simples em bebês, realizada com anestésico tópico e tesoura, em consultório, não se formando nenhuma aderência depois da incisão horizontal.

Marchesan et al. (2012) $)^{9}$ e Gomes et al. (2015) ${ }^{23}$ relataram estudos científicos em crianças maiores, já com complicações maiores advindas da anquiloglossia, dentre elas a deglutição, os movimentos da língua, a fala e a articulação das palavras, exigindo técnica cirúrgica mais específicas, um bom manejo do paciente por parte do odontopediatra e o acompanhamento pós-cirúrgico por equipe multiprofissional, especialmente tratamento com fonoaudiólogo e ortodontia. Brito et al. (2008) $)^{3}$ apontam, ainda, que como adaptações ou compensações durante a fala observa-se a ocorrência da diminuição do espaço entre maxilares, aumento da salivação, movimentos mandibulares excessivos de lateralização e anteriorização, o que pode gerar imprecisão da fala.

A amamentação e a fala são as alterações mais frequentes quando o frênulo da língua se encontra alterado. Segundo os autores, é de extrema relevância o diagnóstico precoce com o objetivo de minimizar as alterações, principalmente para amamentação e funções orofaciais.

Diante dos dados coletados no presente estudo, fica claro que é de extrema importância que as especialidades caminhem juntas, pautadas principalmente no domínio da anatomia orofacial da criança, para proporcionar um planejamento e tratamento integrado, em que o cirurgião-dentista tem papel fundamental. No entanto, é necessário que a amostra seja expandida em trabalhos futuros, a fim de avaliar a maior quantidade possível de profissionais, para que os resultados sejam mais precisos.

\section{CONCLUSÃO}

- Os profissionais avaliados, em sua maioria, não possuem conhecimento sobre o protocolo de avaliação do freio lingual em bebês, bem como da lei que prevê a obrigatoriedade do "Teste da Linguinha";

- Observou-se desconhecimento dos profissionais da área de saúde com respeito a anatomia da boca do bebê, em especial para avaliação do freio lingual, refletindo diretamente num adequado diagnóstico de anquiloglossia e possível indicação cirúrgica;

- Se faz necessária a atualização e treinamento dos profissionais de saúde da rede pública, com enfoque para os aspectos de normalidade da cavidade bucal do bebê, prevenindo desta forma o desmame precoce e ainda a conduta clínica adequada para os casos de anquiloglossia.

\section{REFERÊNCIAS}

1. Ballard JL, Auer CE, Khoury JC. Ankyloglossia: Assessment, Incidence, and Effect of Frenuloplasty on the Breastfeeding Dyad. Pediatrics. 2002; 110(5):63.

2. Braga LAS, Silva J, Pantuzzo CL, Motta AR. Prevalência de alterações de frênulo lingual e suas implicações na fala de escolares. RevCEFAC. 2009;11(3):378-390.

3. Brito SF, Marchesan IQ, Bosco CM, Carrilho ACA, Rehder MI. Frênulo lingual: classificação e conduta segundo ótica fonoaudiológica, odontológica e otorrinolaringológica. Rev CEFAC. 2008;10(3):34351.

4. Martinelli RLC, Marchesan IQ, Berretin-Felix G. Pro- 
tocolo para avaliação do frênulo lingual para bebês: Relação entre aspectos anatômicos e funcionais. Rev CEFAC. 2013;15(3):599-610.

5. Segal LM, Stephenson R, Dawes M, Feldman P. Prevalence, diagnosis, and treatment of ankyloglossia: methodologic review. Can Fam Physician. 2007; 53(6):1027-33.

6. Martinelli RLC, Marchesan IQ, Rodrigues AC, Berretin-Felix G. Protocolo de avaliação do frênulo da língua em bebês. Rev CEFAC. 2012;14(1):138-45.

7. Procópio IMS. Tratamento cirúrgico da anquiloglossia [monografia]. Brasília: Universidade de Brasília; 2014.

8. Buryk M, Bloom D, Shope T. Efficacy of neonatal release of ankyloglossia: a randomized trial. Pediatrics, 2011;128(2):280-8.

9. Marchesan IQ, Martinelli RLC, Gusmão RJ. Frênulo lingual: modificações após frenectomia. J Soc Bras Fonoaudiol. 2012;24(4):409-12.

10. Brasil. Presidência da República. Lei $\mathrm{n}^{\circ} 13.002$, de 20 de junho de 2014. Obriga a realização do Protocolo de Avaliação do Frênulo da Língua em Bebês. Diário Oficial [da União]. Brasília, DF; 23 jun. 2014. Seção 1, p. 4.

11. Cartilha do teste da linguinha: para mamar, falar e viver melhor. São José dos Campos, SP: Pulso Editorial, 2014.

12. Sousa EA. Teste da linguinha agora é obrigatório. [publicação na web]; 2014 acesso em 25 de março de 2015. Disponível em: http://www.redehumanizasus.net/86749-teste-da-linguinha-agora-e-obrigatorio

13. Instituto Brasileiro de Geografia e Estatística. [homepage na internet]. Índice de desenvolvimento humano do município de Maringá-PR[acesso em 25 de março de 2015]. Disponível em: https://cidades. ibge.gov.br/brasil/pr/maringa/pesquisa/37/30255

14. Prefeitura Municipal de Maringá. [página na internet]. Plano Municipal de Saúde de Maringá/Paraná [acesso em 25 de março de 2015]. Disponível em: http://www2.maringa.pr.gov.br/sistema/arquivos/ b65b3c5fcd38.pdf.
15. O'Shea JE, Foster JP, O'Donnell CPF, Breatnach D, Jacobs SE, Todd DA, et al. Frenotomy for tongue-tie in newborn infants. Cochrane Database Syst Rev [Internet]. 2014. [acesso em 2018 jul. 15] Disponível em: https://www.cochranelibrary.com/cdsr/ doi/10.1002/14651858.CD011065.pub2/full

16. Walker RD, Messing S, Rosen-Carole C, Benoit MM. Defining Tip-Frenulum Length for Ankyloglossia and Its Impact on Breastfeeding: A Prospective Cohort Study. Breastfeed Med. 2018;13(3):204-10.

17. Martinelli RLC, Marchesan IQ, Gusmão RJ, Honório HM, Berretin-Felix G. The effects of frenotomy on breastfeeding. J. Appl. Oral Sci. 2015;23(2):153-57.

18. Kupietzky A, Botzer E. Ankyloglossia in the infant and young child: clinical suggestions for diagnosis and management. Pediatr Dent. 2005; 27(1):40-6

19. Walsh J, Tunkel D. Diagnosis and treatment of ankyloglossia in newborns and infants: a review. JAMA. 2017;143(10):1032-39.

20. Silveira LMD, Prade LS, Ruedell AM, Haeffner LSB, Wienmann ARM. Aleitamento materno e sua influência nas habilidades orais de crianças. Revista de Saúde Pública. 2013;47(1):37-43.

21. Kramer MS, Kakuma R. Optimal duration of exclusive breastfeeding. Cochrane Database Syst Rev [Internet]. 2012. [acesso em 2018 jul. 15] Disponível em: https://www.cochranelibrary.com/cdsr/ doi/10.1002/14651858.CD003517.pub2/epdf/fu

22. Melo NSFO, Lima AAS, Fernandes A, Silva RPGVC. Anquiloglossia: relato de caso. RSBO. 2011;8(1):102-7.

23. Gomes E, Araújo FB, Rodrigues JA. Freio lingual: abordagem clínica interdisciplinar da Fonoaudiologia e Odontopediatria. Rev Assoc. Paul. Cir. Dent. 2012;69(1):20-4. 